EGU21-13247, updated on 22 Nov 2021

https://doi.org/10.5194/egusphere-egu21-13247

EGU General Assembly 2021

(c) Author(s) 2021. This work is distributed under

the Creative Commons Attribution 4.0 License.

\title{
Commercial microwave links as rainfall input data in urban hydrological modelling
}

\author{
Greta Cazzaniga ${ }^{1}$, Carlo De Michele ${ }^{1}$, Cristina Deidda ${ }^{1}$, Michele D'Amico ${ }^{2}$, Antonio Ghezzi ${ }^{1}$, and \\ Roberto Nebuloni ${ }^{3}$ \\ ${ }^{1}$ DICA, Politecnico di Milano, Milano, Italy \\ ${ }^{2}$ DEIB, Politecnico di Milano, Milano, Italy \\ ${ }^{3}$ IEIIT, Consiglio Nazionale delle Ricerche, Milano, Italy
}

Many studies in literature have showed that hydrological models are highly sensitive to spatial variability of the rainfall field. Limited and inaccurate rainfall observations can negatively affect flood forecasting and the decision-making processes based on warning system. This problem becomes much more evident in urban catchments which usually covers huge areas and where the runoff process is faster, due to the highly impervious surfaces. Given this, it is a priority to develop always new operational instruments which can improve rainfall data availability and accurately quantify rainfall variability in space. To face this challenge, in the recent years, it has been investigated the use of commercial microwave links (CML) as opportunistic rainfall sensors which could be integrated with traditional rainfall observations in areas lacking sensors. The technique relies on the well-established relationship between CML's signal attenuation and rainfall intensity across the signal propagation path. Here, we assess the operational potential of a CML network, located in the northern area of Lambro river (Lombardia region, Italy). This urbanized region is of great hydrological interest, since it is often subjected to flash floods, hence it requires a robust and accurate warning system. We considered a set of about $80 \mathrm{CMLs}$ distributed quite uniformly over the entire study area and we assessed if and how rainfall data collected by them can improve river discharge predictions. To this aim, we implemented a semi-distributed rainfall-runoff model, which reproduces the river flow at the outlet section in Lesmo (Monza e Brianza), and we fed the hydrological model with CML rainfall data. We tested the use of CML rainfall data as input to the hydrological model. In particular, we used path-averaged rainfall intensities, calculated from CML path attenuation, as point measurements with a weight inversely proportional to CML length. To check the suitability of CML data as input to our urban rainfall-runoff model, we compared the observed river discharge with the predicted one, obtained using different rainfall data layouts. Indeed, we tested CML data but also rain gauges measurements and a combination of CML and rain gauge observations. 